REVIEW

\title{
Aortic sclerosis: not an innocent murmur but a marker of increased cardiovascular risk
}

A K Nightingale, J D Horowitz

See end of article for authors' affiliations

Correspondence to: Dr Angus Nightingale, Department of Cardiology, The Queen Elizabeth Hospital, 28 Woodville Road, Woodville South SA 5011, Australia; drangus@doctors.org.uk

Accepted 15 March 2005 Published Online First 29 March 2005

Heart 2005;91:1389-1393. doi: 10.1136/hrt.2004.057117

\section{Epidemiology}

In the cardiovascular health study, $29 \%$ of the 5621 subjects aged over 65 had aortic sclerosis on echocardiography. ${ }^{1}$ A similar study looking at an older population (mean age 82 years) found a prevalence of $42 \%{ }^{2}$ The prevalence rises further in a higher cardiovascular risk population. In one study, of 425 patients (mean age 68 years) admitted to hospital with chest pain, 50\% were found to have aortic sclerosis. ${ }^{3}$ From this we can conclude that aortic sclerosis is common in the elderly and in a population with risk factors for coronary artery disease (table $\mathrm{I}^{1-9}$ ). One criticism of these studies is that they do not specify whether bicuspid aortic valves were excluded from the study. It is well recognised that bicuspid aortic valves do calcify at an earlier stage and, given a population prevalence of up to $2 \%$, this may have resulted in a slight overestimate of the prevalence of aortic sclerosis in tricuspid aortic valves.

\section{Diagnosis by echocardiography}

A major impediment to the investigation of aortic sclerosis has been the lack of objective methods to quantify the degree of sclerosis. In contrast to aortic stenosis, where velocity is increased through the aortic valve, in aortic sclerosis Doppler measurements are not helpful. There have been attempts to quantify the severity of aortic sclerosis on the basis of the amount of calcification seen on the aortic leaflets in the short axis view on the echocardiogram. Chandra et $a^{3}$ quantified the severity of aortic sclerosis on a scale of 0 to 3: 0, normal (no involvement); 1, mild (minor involvement of one leaflet); 2, moderate (minor involvement of two leaflets or extensive involvement of one leaflet); and 3, severe (extensive involvement of two leaflets or involvements of all three leaflets). Others have used a 1-4 scale. ${ }^{10}$ These methods are still essentially subjective.

\section{DEFINITION AND QUANTIFICATION OF AORTIC SCLEROSIS}

The presence of an aortic systolic murmur is often the first sign that alerts the clinician to the possibility of aortic sclerosis. Unfortunately there are few prospective data on the specificity and sensitivity of this clinical finding compared with the echocardiogram. Aortic sclerosis can be diagnosed on echocardiography by thickening and calcification of one or more leaflets of a tricuspid aortic valve. In contrast with aortic stenosis, leaflet opening is not restricted and the velocity through the aortic valve is $\leqslant 2.5 \mathrm{~m} / \mathrm{s}$. Several prospective population studies have shown that aortic sclerosis is often found in the elderly population.

\section{Tissue quantification (backscatter) echocardiography}

To try to improve on two dimensional echocardiography, we have used ultrasonic tissue characterisation from the backscatter of ultrasound to quantify the degree of aortic sclerosis. ${ }^{11}$ This technique uses tissue Doppler to assess the degree of backscatter from the aortic valve

\footnotetext{
Abbreviations: $A C E$, angiotensin converting enzyme; $\mathrm{ARIC}$, atherosclerosis risk in communities; CT, computed tomography; LIFE, losartan intervention for endpoint reduction in hypertension; SPARC, stroke prevention: assessment of risk in a community
} leaflets in the long axis parasternal view. A 
Table 1 Prevalence of aortic sclerosis based on echocardiographic findings in various study groups

\begin{tabular}{|c|c|c|c|c|c|}
\hline Author & Sample size & Population studied & Age (years) & $\begin{array}{l}\text { Prevalence of } \\
\text { aortic sclerosis }\end{array}$ & Relative risk of sclerosis/event \\
\hline OHo $^{1}$ & 5621 & $\begin{array}{l}\text { Population study, cardiovascular health } \\
\text { study }\end{array}$ & $>65$, mean $75(6)$ & $29 \%$ & $1.66 /$ death \\
\hline Aronow $^{2}$ & 2358 & Population study & Mean 82 & $42 \%$ & $1.76 /$ new coronary event \\
\hline Taylor $^{4}$ & 2279 & $\begin{array}{l}\text { Population study, ARIC (African American) } \\
\text { substudy }\end{array}$ & $65-74$ & $18.6 \%$ & $\begin{array}{l}3.8 / \mathrm{Ml} \text { or fatal coronary } \\
\text { disease }\end{array}$ \\
\hline Olsen 5,6 & 960 & Hypertension with ECG LVH, LIFE substudy & Mean 66 & $40.4 \%$ & $\begin{array}{l}2.0 / \text { composite cardiac end } \\
\text { point }\end{array}$ \\
\hline Chandra $^{3}$ & 425 & Admissions with chest pain & Mean 68 & $50 \%$ & 2.4/cardiovascular event \\
\hline Agmon $^{7}$ & 381 & Population study & Mean 67 , range $51-101$ & $36.7 \%$ & ND \\
\hline Yamaura $^{8}$ & 252 & Healthy subjects & $25-65$ & $11 \%$ & ND \\
\hline Poggianti ${ }^{9}$ & 102 & $\begin{array}{l}\text { Suspected coronary disease awaiting stress } \\
\text { echocardiography }\end{array}$ & $63.5(9.7)$ & $34 \%$ & ND \\
\hline
\end{tabular}

ARIC, atherosclerosis risk in communities; LIFE, losartan intervention for endpoint reduction in hypertension; LVH, left ventricular hypertrophy; MI, myocardial infarction; ND, not determined.

higher backscatter score is seen from valves that are sclerotic than from non-sclerotic valves. In addition, backscatter score is increased further in stenotic valves. Thus, this technique reflects the impact of combined thickening, fibrosis, and calcification of the valve matrix in association with sclerosis (figs 1 and 2). This has two important implications. Firstly, this technology is available on most recent cardiac ultrasound packages for offline analysis. This should enable better assessment of aortic sclerosis, as it provides objective, quantitative evidence of aortic valve thickening and calcification. Secondly, as backscatter measures are readily reproducible, this opens up the possibility to perform longitudinal studies to document the natural history of aortic sclerosis and to assess interventions aimed at preventing progression of sclerosis to stenosis.

\section{Aortic sclerosis and calcification}

Although two dimensional echocardiography has been the mainstay of diagnosis of aortic sclerosis, in particular because of its utility in population based studies, other imaging techniques have been proposed. Both electron beam computed tomography (CT) and more recently multidetector CT can quantify the degree of calcification of the aortic valve (fig 3). As well as assessing aortic valve calcification, these and other techniques can detect calcium in the mitral annulus, ${ }^{12}{ }^{13}$ aortic arch, ${ }^{14}$ abdominal aorta ${ }^{15}{ }^{16}$ and coronary arteries. ${ }^{17}$ Many studies have shown the prognostic significance of this vascular calcification in asymptomatic populations $^{13}$ and in selected patient groups such as those with chronic heart failure ${ }^{15}$ diabetes mellitus, ${ }^{18}$ and chronic renal failure. ${ }^{19}$ Although the finding of calcification has clear prognostic implications, it is a relatively late event. In a substudy of the Framingham population, Walsh et al ${ }^{17}$ used electron beam CT to assess aortic valve and coronary calcification. They found that only $20 \%$ of patients with aortic sclerosis (based on two dimensional echocardiography) had calcification of the aortic valve. Therefore, these methods, although specific, lack sensitivity in detecting early aortic valve disease. With current technologies, it seems that echocardiography, especially with tissue characterisation of the aortic valve by backscatter, is the most sensitive way of detecting aortic sclerosis.

\section{PROGRESSION OF AORTIC SCLEROSIS}

The traditional view was that, although aortic sclerosis may have been fairly common, it was of no great significance. The main focus was on the risk of endocarditis or embolism from a sclerotic valve. No prospective data have quantified this risk. Of more concern has been the progression of aortic sclerosis to stenosis. The literature lacks any prospective data on this as well. The LIFE (losartan intervention for endpoint reduction in hypertension) substudy gives some insight into the effects of age on development and progression of aortic sclerosis. ${ }^{5}$ In both treatment groups, the rate of progression from normal aortic valve to sclerosis was around $24 \%$ over four years. Although the rate of progression in a population is unknown, the incidence of aortic sclerosis increases with age as table 1 shows. Indeed, further quantification of the degree of aortic sclerosis by ultrasound backscatter shows a strong correlation between age and backscatter on univariate analysis (fig 4). There are at least two components to the effect of aging: firstly, mechanical stress on the valve increases with time, and secondly, exposure to risk factors over time is important.

\section{Progression of sclerosis to stenosis}

Most of the studies on aortic stenosis have identified an asymptomatic population with some degree of aortic stenosis (restricted leaflet opening and an aortic jet velocity of $\geqslant 2.5 \mathrm{~m} / \mathrm{s}$ at baseline). ${ }^{20}$ Two retrospective studies have looked at progression to aortic stenosis. Cosmi et $a l^{21}$ analysed data from 2131 patients with aortic valve thickening (defined as focal or diffuse leaflet thickening or calcification, normal valve excursion, and peak Doppler flow velocity of $<2 \mathrm{~m} / \mathrm{s}$ )
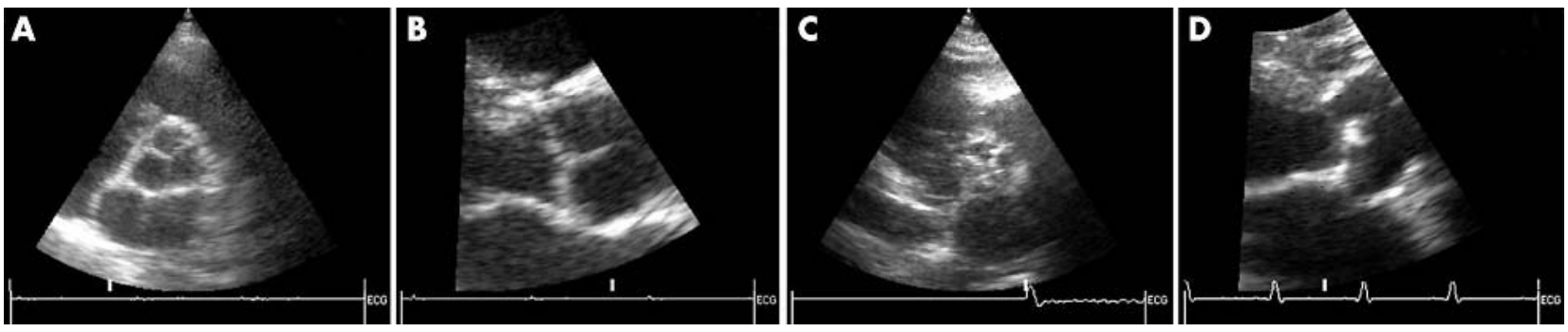

Figure 1 Echocardiographic images of aortic sclerosis showing (A) a normal valve in the parasternal short axis and (B) a zoom view in the parasternal long axis, compared with (C) a sclerotic valve in the short axis and (D) the long axis. 


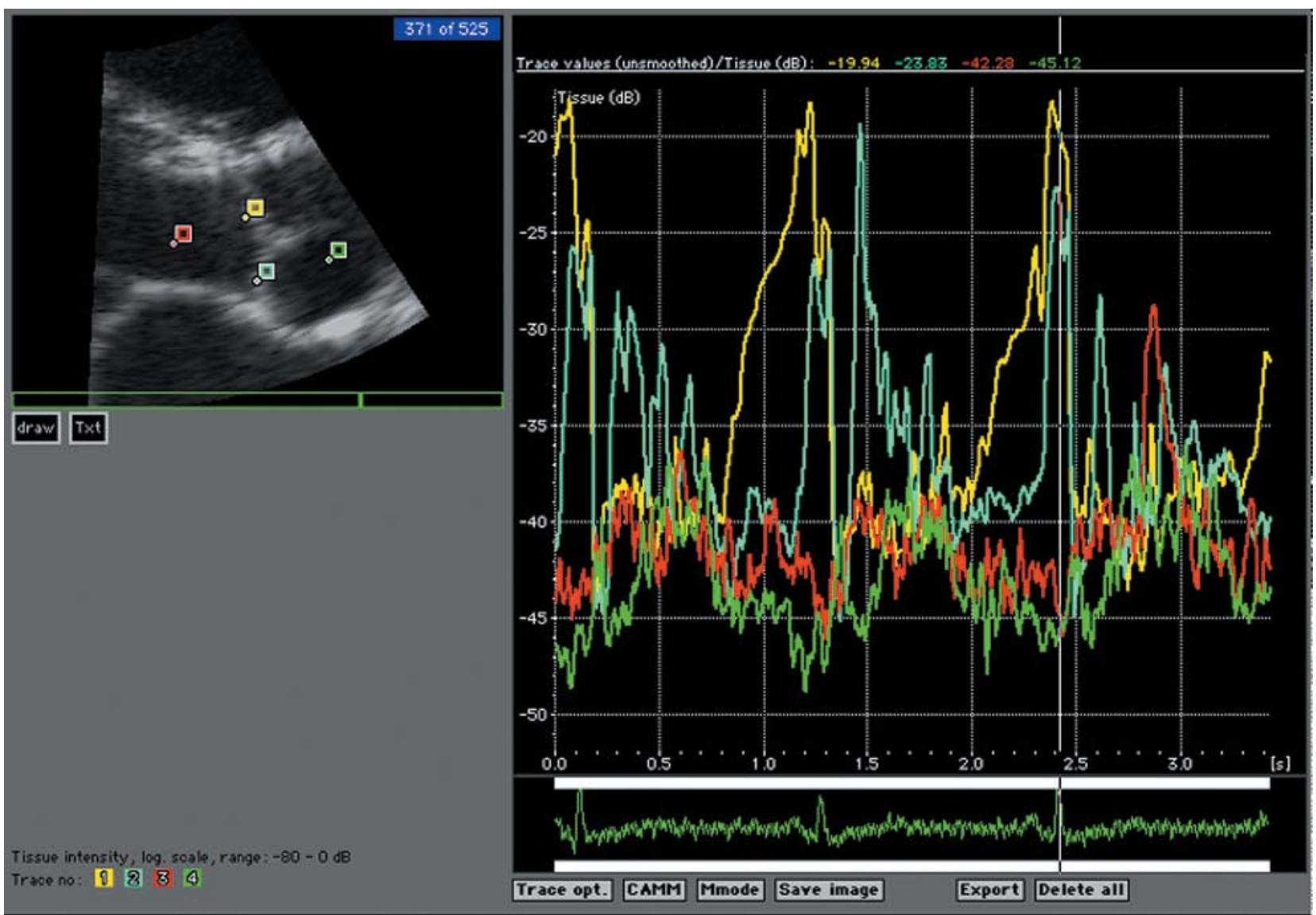

Figure 2 The backscatter score is measured by tissue quantification (Vingmed Echopac; Vingmed, Horten, Norway) in the zoom view of the parasternal long axis. ${ }^{5}$

who had at least one year of follow up. Of these, 338 (15.9\%) developed aortic stenosis: 223 developed mild, 61 moderate, and 54 severe aortic stenosis over a mean follow up of 7.4 years. This study therefore suggested that aortic sclerosis progressed to clinically significant (moderate or severe) aortic stenosis in $5.4 \%$ of patients with aortic sclerosis over a period of seven years. Importantly, all patients with aortic stenosis (with one exception) started with aortic sclerosis. Although these findings have established that aortic sclerosis may progress to stenosis, they do not establish the rate of progression in the group as a whole. Faggiano et $a^{22}$ studied 400 patients with abnormal aortic valves at baseline. Of these, 131 went on to develop some degree of aortic stenosis over a mean period of four years. This suggests that aortic sclerosis may be important as a precursor to aortic stenosis.

Further evidence regarding the natural history of aortic stenosis is provided by a recent publication from Rosenhek et al, ${ }^{10}$ who investigated 176 asymptomatic patients with mild to moderate aortic stenosis. Those authors showed that this group of patients (who have previously been thought to have a good prognosis) have rapid progression of valve disease, with $46 \%$ developing severe aortic stenosis over five years, and increased mortality. Event-free survival of patients with moderate or severe calcification was worse than that of patients without significant calcification. In fact, the degree of aortic valve calcification was the most powerful predictor of outcome. This also highlights the importance of aortic valve calcification early in the disease process.

\section{Interventions to delay progression}

Having established the importance of aortic valve sclerosis, is there any evidence that this can be reduced and thereby improve outcomes? There are relatively few data on early aortic valve disease. Two studies have included a population without significant aortic stenosis. Pohle $e t a^{23}$ used electron beam CT to study patients with aortic valve calcification. No echocardiographic data were provided on these patients, so we cannot determine whether they had aortic sclerosis or stenosis (although the presence of calcification is more likely to indicate relatively advanced disease). ${ }^{17}$ However, they did show that calcification of the aortic valve progressed more rapidly in patients with low density lipoprotein cholesterol of $>3.37 \mathrm{mmol} / \mathrm{l}$. Similarly, Aronow et al ${ }^{24}$ retrospectively analysed data from 180 patients with a peak gradient of $10-25 \mathrm{~mm} \mathrm{Hg}$ across the aortic valve. They found that factors that predicted an increase in gradient over two years were male sex, smoking, hypertension, diabetes mellitus, and hypercholesterolaemia. Of particular interest was the finding that hydroxymethyl glutaryl coenzyme A (HMG-CoA) reductase inhibitor usage was associated with decreased likelihood of progression. This was further supported from retrospective data from electron beam CT examination of progression of aortic valve calcification. ${ }^{25}$

The pathophysiology of aortic stenosis suggests that angiotensin converting enzyme (ACE) inhibitors may be of value, as ACE is co-localised in the lesion on the valve and angiotensin II may mediate fibrotic and apoptotic changes in the valve. Although ACE inhibitors are relatively contraindicated in advanced aortic stenosis, early use of ACE inhibitors may delay progression of sclerosis to stenosis. The aortic sclerosis substudy of the LIFE study gives important insights into the progression of aortic valve disease. ${ }^{5}$ This study compared two antihypertensive regimens based on atenolol and losartan, respectively. In this study, 960 patients 

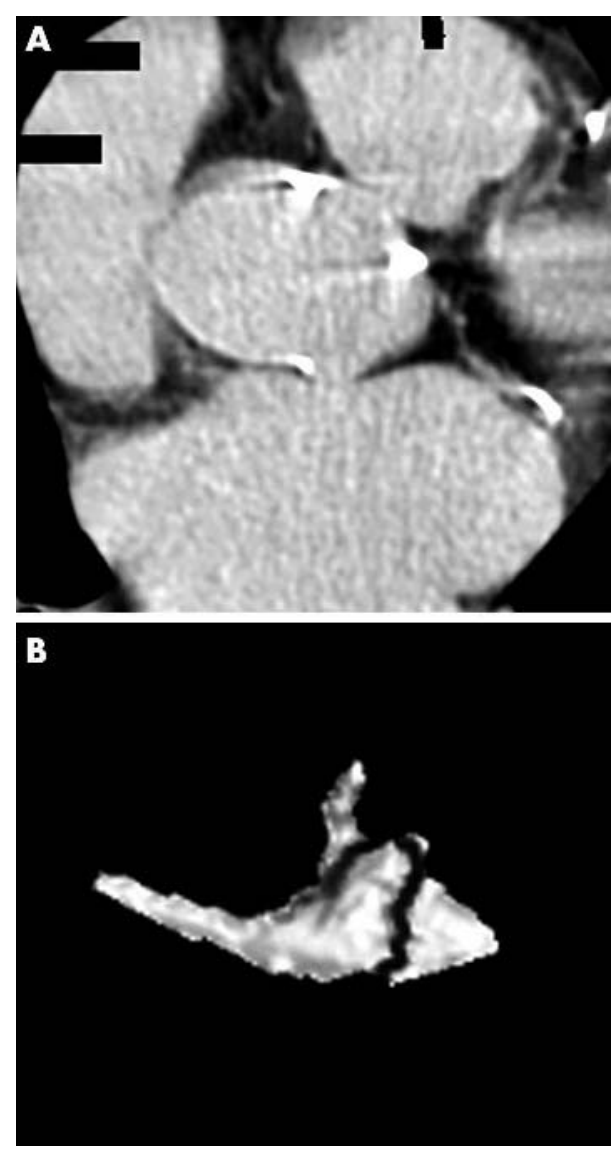

Figure 3 (A) Multislice computed tomography (CT) can also show calcification of the aortic valve in the short axis. (B) This can be reconstructed to quantify the degree of calcification.

aged 50-80 years with ECG evidence of left ventricular hypertrophy underwent echocardiography at baseline and one, two, and fours years. The prevalence of aortic sclerosis increased from $40 \%$ at baseline to $64 \%$ at four years of follow up. Both antihypertensive regimens were similarly unsuccessful in preventing progression of aortic valve disease (from normal to sclerosis and sclerosis to stenosis). The lack of benefit of losartan relative to atenolol argues against the importance of angiotensin II in the progression of aortic valve disease. However, there are certain caveats in making this judgement. Firstly, there was no placebo group and therefore we can only say that the rate of progression was similar in both treatment groups. Secondly, the visual grading of aortic valve morphology into normal, sclerotic, or stenotic means that this is a relatively insensitive method for assessing progression of stenosis.

\section{AORTIC SCLEROSIS AS A MARKER OF INCREASED CARDIOVASCULAR RISK}

Two large studies showed that the presence of aortic sclerosis was independently associated with an increased risk of death from cardiovascular causes, for example, with a relative risk of death of 1.66 (95\% confidence interval (CI) 1.23 to 2.23$)^{1}$ or of a new coronary event of 1.76 (95\% CI 1.52 to 2.03$).{ }^{2}$ In addition, the patients admitted with chest pain and aortic sclerosis had a higher incidence of cardiovascular events $(16.8 \% v 7.1 \%)$ and worse event-free survival than did those without aortic sclerosis. ${ }^{3}$ It has therefore been proposed that aortic sclerosis gives a "window to the coronary arteries" (without the need for an angiogram). ${ }^{26}$

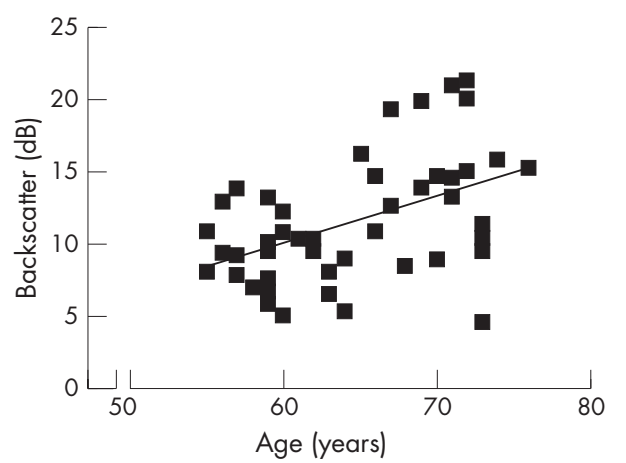

Figure 4 Relation between backscatter score (severity of aortic sclerosis) and age in a healthy population. Linear regression, $p=0.0006, r^{2}=0.22$.

These findings have two implications. Firstly, there is a pathogenic link between aortic sclerosis and acute coronary syndromes that is beyond that of shared coronary risk factors. Secondly, the finding of aortic sclerosis is an incremental risk above conventional risk factors. Thus, the finding of an aortic systolic murmur on routine examination may be regarded as a "better earlobe crease". We will examine this in more detail.

\section{Possible mechanisms of increased risk}

It is crucial to understand more about the mechanisms involved in the development and progression of aortic sclerosis and the precise reason why it acts as a marker of cardiovascular risk. The factors implicated so far in the pathogenesis of aortic sclerosis bear a striking resemblance to those for coronary atherosclerosis. These include male sex, smoking, hypertension, hypercholesterolaemia, and diabetes mellitus. ${ }^{27}$ More specifically, renal disease and disorders of calcium and phosphate metabolism clearly accelerate the process. Histological work has shown that the changes correlating to aortic sclerosis are lipid deposition, macrophage and $\mathrm{T}$ cell infiltration, basement membrane disruption, stippled mineralisation, and small numbers of smooth muscle cells. ${ }^{28}$ These have suggested that low areas of shear stress on the valve interact with an active inflammatory response similar to that seen in atherosclerosis.

There is now further strong evidence for the role of inflammation in aortic sclerosis. Chandra et al showed that patients with aortic sclerosis had a rise of $\mathrm{C}$ reactive protein concentration. Patients with moderate to severe aortic sclerosis had the highest concentrations of $\mathrm{C}$ reactive protein. In addition, patients with moderate to severe aortic sclerosis and the highest tertile of $\mathrm{C}$ reactive protein had a fivefold increase in adverse events at one year compared with those with the lowest tertile of $\mathrm{C}$ reactive protein. Those authors concluded that the adverse cardiovascular outcomes of patients with aortic sclerosis are attributable to the effects of coronary artery disease and inflammation rather than to the valve disease itself. Similarly, but in a lower risk population, the SPARC (stroke prevention: assessment of risk in a community) study showed a weak association between $\mathrm{C}$ reactive protein and aortic sclerosis but this was not independent of other risk factors. ${ }^{7}$ Importantly, blood counts, fibrinogen, and Chlamydia pneumoniae seropositivity were not associated with aortic sclerosis.

Is the aortic valve just a mirror of what is occurring in the coronary arteries? This is difficult to believe, as fewer than $50 \%$ of patients needing aortic valve replacement require simultaneous coronary artery bypass surgery. ${ }^{10}$ We have previously shown that patients with aortic stenosis have platelet dysfunction, with platelet hyperaggregability and 
impaired responsiveness to nitric oxide. ${ }^{29}$ This platelet dysfunction was unrelated to the presence of concomitant coronary artery disease. This suggests that other mechanisms are involved or that susceptibility of the aortic valve or coronary arteries is altered in patients. Poggianti et $a l^{9}$ investigated whether in high risk patients (referred for a stress echocardiography) aortic sclerosis was related to endothelial function (measured by post-ischaemic dilatation of the brachial artery). They found that $32 \%$ of patients had aortic sclerosis. Those with aortic sclerosis had a significantly reduced endothelium dependent flow mediated dilatation of the brachial artery during reactive hyperaemia. The two groups did not differ significantly with regard to the presence or severity of coronary artery disease, although the study was not powered to investigate this specifically. A similar study looked at carotid intima-media thickness in 252 healthy subjects aged 25-65 years old; $11 \%$ of these had aortic sclerosis. Carotid intima-media thickness was significantly greater in those with aortic sclerosis. ${ }^{8}$

In the cardiovascular health study, the rate of death from any cause or death from cardiovascular cause of patients with aortic sclerosis was twice that of patients with a normal aortic valve. ${ }^{1}$ This ratio persisted after adjustment for age and sex and appeared to be independent of baseline risk factors for aortic sclerosis. The increased risk was most apparent in patients without clinically evident coronary disease at entry into the study. This suggests that aortic sclerosis not only is a marker of the presence of coronary artery disease but also reflects the sum of a number of risk factors potentially reflecting susceptibility to coronary thrombosis. Further evidence comes from data on African Americans in the Jackson cohort of the ARIC (atherosclerosis risk in communities) study. In 2279 middle aged African Americans, the presence of aortic sclerosis conferred a hazard ratio of 3.8 for myocardial infarction or fatal coronary heart disease after adjustment for multiple risk factors. ${ }^{4}$ The recent LIFE substudy on aortic sclerosis also confirmed a higher risk with twice the risk of serious cardiovascular events in patients with aortic sclerosis. ${ }^{\circ}$

Thus, aortic sclerosis is a common condition, especially in the elderly population, and can no longer be viewed as an innocent murmur, as it carries a 50\% increase in risk of cardiac death or myocardial infarction. Whatever the mechanism of this association, it is easily detectable and new tools available in most echocardiography laboratories facilitate its quantification. The finding of aortic sclerosis should be reported on the echocardiogram (although many units do not do this due to lack of quantification to support the visual appearance) and alert the physician to increased cardiac risk. We await with interest randomised prospective studies that will show whether the rate of aortic sclerosis progressing to stenosis can be altered and whether the presence of aortic sclerosis can be therapeutically dissociated from risk of acute coronary syndromes.

\section{ACKNOWLEDGEMENTS}

Computed tomograms in fig 3 kindly provided by Dr G Morgan Hughes, South West Cardiothoracic Centre Plymouth, UK. Unpublished data in fig 4 provided by D Ngo, Adelaide, Australia, 2005.

\section{Authors' affiliations}

A K Nightingale, J D Horowitz, Department of Cardiology, The Queen Elizabeth Hospital, The University of Adelaide, Adelaide, Australia

Both authors fulfil the criteria for authorship and have no competing interests to declare.

\section{REFERENCES}

1 Otto CM, Lind BK, Kitzman DW, et al. The cardiovascular HS. Association of aortic-valve sclerosis with cardiovascular mortality and morbidity in the elderly. N Engl J Med 1999;341:142-7.

2 Aronow WS, Ahn C, Shirani J, et al. Comparison of frequency of new coronary events in older subjects with and without valvular aortic sclerosis. Am J Cardiol 1999;83:599-600.

3 Chandra HR, Goldstein JA, Choudhary N, et al. Adverse outcome in aortic sclerosis is associated with coronary artery disease and inflammation. J Am Coll Cardiol 2004:43:169-75.

4 Taylor J, Clark BL, Garrison RJ, et al. Relation of aortic valve sclerosis to risk of coronary heart disease in African-Americans. Am J Cardiol 2005;95:401-4.

5 Olsen MH, Wachtell K, Bella JN, et al. Effect of losartan versus atenolol on aortic valve sclerosis (a LIFE substudy). Am J Cardiol 2004;94:1076-80.

6 Olsen MH, Wachtell K, Bella JN, et al. Aortic valve sclerosis relates to cardiovascular events in patients with hypertension (a LIFE substudy). Am J Cardiol 2005;95:132-6.

7 Agmon Y, Khandheria BK, Jamil Tajik A, et al. Inflammation, infection, and aortic valve sclerosis: insights from the Olmsted County (Minnesota) population. Atherosclerosis 2004; 174:337-42.

8 Yamaura Y, Nishida T, Watanabe N, et al. Relation of aortic valve sclerosis to carotid artery intima-media thickening in healthy subjects. Am J Cardiol 2004; $94: 837-9$.

9 Poggianti E, Venneri L, Chubuchny V, et al. Aortic valve sclerosis is associated with systemic endothelial dysfunction. J Am Coll Cardiol 2003:41:136-41.

10 Rosenhek R, Klaar U, Schemper M, et al. Mild and moderate aortic stenosis: natural history and risk stratification by echocardiography. Eur Heart $J$ 2004;25: 199-205.

11 Ngo DTM, Wuttke RD, Turner S, et al. Quantitative assessment of aortic sclerosis using ultrasonic backscatter. J Am Soc Echocardiogr 2004; 17:1123-30.

12 Fox E, Harkins D, Taylor H, et al. Epidemiology of mitral annular calcification and its predictive value for coronary events in African Americans: The Jackson cohort of the atherosclerotic risk in communities study. Am Heart $J$ 2004; 148:979-84

13 Fox CS, Vasan RS, Parise $\mathrm{H}$, et al. Mitral annular calcification predicts cardiovascular morbidity and mortality: the Framingham heart study. Circulation 2003;107:1492-6

14 Iribarren C, Sidney S, Sternfeld B, et al. Calcification of the aortic arch: risk factors and association with coronary heart disease, stroke, and peripheral vascular disease. JAMA 2000;283:2810-5.

15 Walsh C, Cupples L, Levy D, et al. Abdominal aortic calcific deposits are associated with increased risk for congestive heart failure: the Framingham heart study. Am Heart J 2002; 144:733-9.

16 Wilson PWF, Kauppila LI, O'Donnell CJ, et al. Abdominal aortic calcific deposits are an important predictor of vascular morbidity and mortality. Circulation 2001;103:1529-34.

17 Walsh CR, Larson MG, Kupka MJ, et al. Association of aortic valve calcium detected by electron beam computed tomography with echocardiographic aortic valve disease and with calcium deposits in the coronary arteries and thoracic aorta. Am J Cardiol 2004;93:421-5.

18 Lehto S, Niskanen L, Suhonen M, et al. Medial artery calcification: a neglected harbinger of cardiovascular complications in non-insulin-dependent diabetes mellitus. Arterioscler Thromb Vasc Biol 1996;16:978-83.

19 Blacher J, Guerin AP, Pannier B, et al. Arterial calcifications, arterial stiffness, and cardiovascular risk in end-stage renal disease. Hypertension $2001 ; 38: 938-42$

20 Otto CM, Burwash IG, Legget ME, et al. Prospective study of asymptomatic valvular aortic stenosis : clinical, echocardiographic, and exercise predictors of outcome. Circulation 1997;95:2262-70.

21 Cosmi JE, Kort S, Tunick PA, et al. The risk of the development of aortic stenosis in patients with "benign" aortic valve thickening. Arch Intern Med 2002; 162:2345-7.

22 Faggiano $\mathbf{P}$, Antonini-Canterin F, Erlicher A, et al. Progression of aortic valve sclerosis to aortic stenosis. Am J Cardiol 2003;91:99-101.

23 Pohle K, Maffert R, Ropers D, et al. Progression of aortic valve calcification: association with coronary atherosclerosis and cardiovascular risk factors. Circulation 2001;104:1927-32.

24 Aronow WS, Ahn C, Kronzon I, et al. Association of coronary risk factors and use of statins with progression of mild valvular aortic stenosis in older persons. Am J Cardiol 2001;88:693-5.

25 Shavelle DM, Takasu J, Budoff MJ, et al. HMG CoA reductase inhibitor (statin) and aortic valve calcium. Lancet 2002;359:1125-6.

26 Carabello BA. Aortic sclerosis: a window to the coronary arteries? N Engl J Med 1999;341:193-5.

27 Stewart MD, Siscovick MD, Lind MS. Clinical factors associated with calcific aortic valve disease. J Am Coll Cardiol 1997:29:630-4.

28 Otto CM, Kuusisto J, Reichenbach DD, et al. Characterization of the early lesion of 'degenerative' valvular aortic stenosis: histological and immunohistochemical studies. Circulation 1994;90:844-53.

29 Chirkov YY, Holmes AS, Willoughby SR, et al. Association of aortic stenosis with platelet hyperaggregability and impaired responsiveness to nitric oxide. Am J Cardiol 2002;90:551-4. 